\title{
Impact of COVID-19 Lockdown and Atmospheric Circulation on the Air Quality in Wuhan During Early 2020
}

\author{
Youyong $\mathrm{Xie}^{1}$ and Xiefei $\mathrm{Zhi}^{1,2, *}$ \\ ${ }^{1}$ School of Atmospheric Sciences, Nanjing University of Information Science \& Technology, Nanjing \\ 210044, China \\ ${ }^{2}$ WeatherOnline Institute of Meteorological Applications, Wuxi 214000, China
}

\begin{abstract}
Previous studies indicated that the air quality was improved in Wuhan during COVID-19 lockdown. However, the impact of atmospheric general circulation on the changes of air quality has not been taken into account. The present study aims to discuss the improvement of air quality in Wuhan and its possible reasons during COVID-19 lockdown. The results showed that all air pollutants except $\mathrm{O}_{3}$ decreased in Wuhan during early 2020. The occurrence days of A, C, W and NW types' circulation pattern during early 2020 are more than those during the same period of 1979-2020. The occurrence days of SW type's circulation pattern is slightly less than those during early 1979-2020. With more occurrence days of these dominant atmospheric circulation patterns, the number of polluted days could rise in Wuhan during early 2020. Nevertheless, this scenario didn't occur. The COVID-19 lockdown did improve the air quality in Wuhan during early 2020.
\end{abstract}

\section{Introduction}

The Coronavirus Disease 2019 (COVID-19) pandemic has swept the world, bringing great changes to many countries and people. In early 2020, COVID-19 broke out in Wuhan, China. In order to curb the outbreak of COVID-19, strict and efficient measures were taken. The COVID-19 lockdown were mandated from January 23 to April 8, 2020 in Wuhan (January 23 to April 8; hereafter LDJA). These measures included traffic restrictions, closing a part of industries and business, and bans on public meetings, etc. In fact, the pandemic lockdown measures were taken in many countries worldwide during the past one and half years. According to IQAir's 2020 World Air Quality Report, the human-related emissions from industry and transport decreased during lockdown, and $65 \%$ of global cities analyzed reported better air quality in 2020 compared to 2019 . Some $84 \%$ of nations surveyed experienced air quality enhancements overall.

During COVID-19 lockdown, the concentration of air pollutants fell. Many studies [1-5] indicate that except for $\mathrm{O}_{3}$, the concentration of air pollutants, e.g. $\mathrm{NO}_{2}, \mathrm{PM}_{2.5}$ and $\mathrm{PM}_{10}$, in

* Corresponding author: zhi@nuist.edu.cn 
Wuhan, and in many other cities of China decreased substantially during COVID-19 lockdown, compared to previous years.

Generally speaking, there are many factors which may influence the concentration of local air pollutants and air quality. At least, anthropogenic factors and atmospheric factors should be taken into account. Anthropogenic factors are the important factors of local pollutant concentration changes. However, there are many factors in atmosphere which play an important role in air quality variation. For instance, temperature inversion and mixing height is critical indicators for air pollution in hot arid climate [6]; stable ground inversion layer could make air pollution become more serious with the discharge of pollutants [7]. To take a step further, the inversion is associated with wind speed maxima and strong vertical wind speed gradients [6]. Furthermore, $\mathrm{NO}_{\mathrm{x}}(\mathrm{CO})$ is positively (negatively) associated with relative humidity from January to April in Addis Ababa, Ethiopia [8].

Additionally, atmospheric general circulation also has a vital influence on the concentration of pollutants. Due to the strong East Asian trough, meridional circulation prevails in Asia and the concentration of pollutants in Northwest China is reduced [9]. In order to study how the different circulations affect the concentration of air pollutants, a number of studies have developed approaches to classify the atmospheric circulation patterns and applied to practice, such as the configuration of pressure systems and the relative position of study area [10] and some subjective classification methods [11-12]. Lamb-Jenkinson method [13-15] is also used to classify the surface circulation patterns. It is applied to analyze the major circulation patterns of Wuhan and discuss the relationship between the circulation patterns and pollutant concentrations [13]. The same method is used to study the relationship between the circulation patterns and air quality in ChengduChongqing areas [14] and southwestern Russia [15].

Nevertheless, previous studies [1-5] only focus on the phenomenon that the concentrations of air pollutants decline, or they attribute the cause of air pollutant concentration reduction to any factors but atmospheric general circulation. Wang et al.[4] found that low surface wind speed, low vertical wind shear, high vertical potential temperature difference and high surface relative humidity are not favorable for the diffusion of surface air pollutants during COVID-19 lockdown. Sulaymon et al.[5] studied the relationship between the concentrations of pollutants and wind directions, and found that the highest concentrations of $\mathrm{NO}_{2}, \mathrm{PM}_{2.5}$ and $\mathrm{PM}_{10}$ are associated with the southwesterly wind during COVID-19 lockdown. In this study, we aim to discuss the relationship between the improvement of air quality and atmospheric general circulation in Wuhan during LDJA. The result will help to understand the impact of the COVID-19 lockdown on the air quality.

\section{Data and methods}

Gridded daily mean sea level pressure data at 00:00 (UTC) was obtained from ERA5 developed by European Centre for Medium-Range Weather Forecasts (ECMWF). The horizontal resolution of the data is $2.5^{\circ} \times 2.5^{\circ}$. The daily data of air quality index (AQI) and individual air quality index (IAQI) were obtained from Wuhan Ecology and Environment Bureau (http://hbj.wuhan.gov.cn/hjsj/hjsjcx/). The AQI and IAQIs of $\mathrm{SO}_{2}, \mathrm{NO}_{2}, \mathrm{PM}_{10}, \mathrm{CO}$, $\mathrm{O}_{3}$, and $\mathrm{PM}_{2.5}$ during LDJA from 2016 to 2020 are chosen. The atmospheric environmental monitoring site is set as City Proper. Hereafter, the "Wuhan" refers to City Proper of Wuhan.

AQI and IAQI are used to describe the degree of air pollution and the main components of air pollution. According to Technical Regulation on Ambient Air Quality Index (on trial)[16], when AQI is greater than 100 or IAQI is greater than 100 , it is recorded as a 
polluted day with varying degrees. In present study, the degree of pollution is not taken into account. If AQI $>100$ or IAQI $>100$, then a polluted day is recorded.

Lamb-Jenkinson method is used to classify the circulation patterns. The circulation patterns are divided into 4 categories and 27 subcategories (Table 1) by using 3 basic variables over the study area: the direction of mean flow, the strength of mean flow $(|\mathbf{u}|)$ and the vorticity $(\zeta$ ). This method has long been effective in the classification of atmospheric circulation patterns [13-15]. After Chen et al.[13], statistical analysis method and response indexes are used to study the relationship between the improvement of air quality and the surface circulation situation in Wuhan during LDJA.

Table 1. Lamb-Jenkinson circulation type classification scheme. The first column gives 8 advection types based on wind direction. In the second column, A (C) type is anticyclonic (cyclonic) circulation pattern. 16 hybrid types are shown in the third column. U type represents a pattern with weak pressure gradient and vorticity in the fourth column.

\begin{tabular}{cccc}
\hline$|\zeta| \leq|\mathbf{u}|$ & $|\zeta| \geq 2|\mathbf{u}|$ & $|\mathbf{u}| \leq|\zeta| \leq 2|\mathbf{u}|$ & $|\mathbf{u}|<6$ and $|\zeta|<6$ \\
\hline N NE E & A & AN ANE AE CN CNE CE & U \\
SE S SW & C & ASE AS ASW CSE CS CSW & \\
W NW & & AW ANW CW CNW & \\
\hline
\end{tabular}

\section{Change of air quality}

In this section, the fact that the concentrations of air pollutants decreased during LDJA in 2020 is verified. The anomalous percentage is used to analyze the difference of air quality between LDJA in 2020 and the same period in 2016-2019. The anomalous percentage of air pollutants is defined as follow:

$$
x=\frac{x-\bar{x}}{\bar{x}}
$$

Where the $x$ ' denotes the anomalous percentage of air pollutants, $x$ signifies the AQI or IAQIs of pollutants during LDJA in 2020, and $\bar{x}$ is the average of AQI or IAQI of each pollutant during LDJA from 2016 to 2020.

Fig. 1 shows that the anomalous percentage of all air pollutants except $\mathrm{O}_{3}$ are generally negative. The concentrations of pollutants during LDJA in 2020 are indeed lower than those during the same period in previous years. In fact, there are only two polluted days during LDJA in 2020, i.e., January 23 and February 5. Additionally, when the anomalous percentage of other pollutants, e.g., $\mathrm{PM}_{2.5}$ and $\mathrm{SO}_{2}$, is negative, the anomalous percentage of $\mathrm{O}_{3}$ is positive, which is found by other studies $[2-4,17]$ as well. This phenomenon may be caused by reductions in $\mathrm{NO}_{\mathrm{x}}$ emissions, which has weakened the $\mathrm{NO}_{\mathrm{x}}$ titration effect resulting in increases in $\mathrm{O}_{3}$ concentration [17-18].

\section{Major circulation patterns of Wuhan during LDJA}

In order to study the influence of atmospheric general circulation during LDJA, we have analyzed the characteristics of major circulation patterns of Wuhan.

The occurrence frequency of circulation patterns is defined as the ratio of the number of days when circulation patterns occur to the total number of days during LDJA. The three circulation patterns with the highest occurrence frequency are defined as the major circulation patterns of Wuhan during LDJA. 


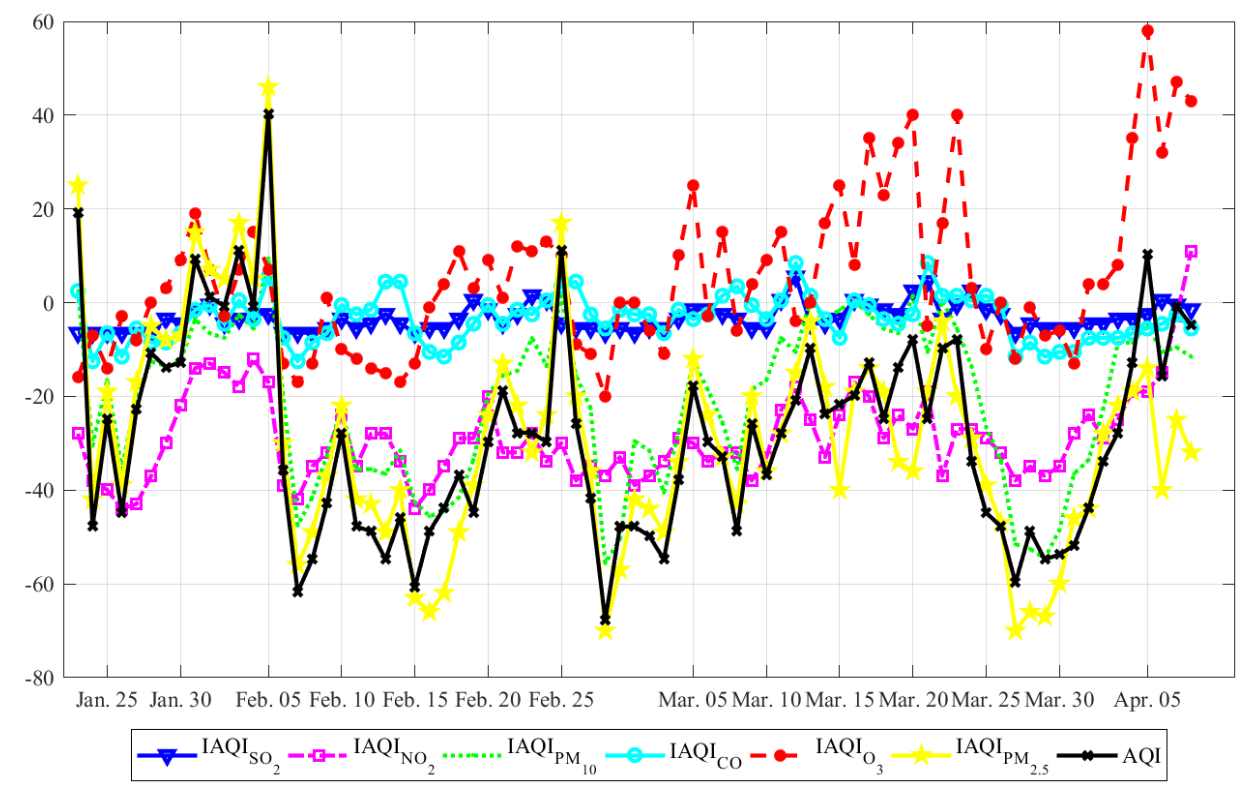

Fig. 1. Anomalous percentage of AQI and IAQIs of $\mathrm{SO}_{2}, \mathrm{NO}_{2}, \mathrm{PM}_{10}, \mathrm{CO}, \mathrm{O}_{3}$, and $\mathrm{PM}_{2.5}$ during LDJA in 2020

During the period of LDJA from 1979 to 2020 (Table 2), there are three circulation patterns with the highest occurrence frequency in Wuhan: A, SW and ASW types (Figs. 2ac). The occurrence frequency is $46.18 \%, 5.34 \%$ and $4.71 \%$, respectively. During LDJA from 2016 to 2020 (Table 3), the three circulation patterns with the highest occurrence frequency in Wuhan are A, C and SW types (Figs. 2a, e and b). The occurrence frequency is $51.31 \%, 5.50 \%$ and $5.24 \%$, respectively.

During LDJA, A type is the most frequent type, and the occurrence frequency is more than $45 \%$, which was consistent with the analysis of Chen et al. [13]. This shows that Wuhan is mainly controlled by ground high pressure weather systems during LDJA. The air quality of Wuhan is the worst in winter, and high temperature, low humidity and breeze are favorable to the accumulation of pollutants in Wuhan during winter season [19]. And the stable atmospheric stratification with low wind speed in Wuhan during winter is generally controlled by the cold high pressure (the same as A type) [20].

Table 2. Number of days and occurrence frequency of circulation patterns during $1979-2020$.

\begin{tabular}{ccc}
\hline Pattern & Days & Occurrence frequency [\%] \\
\hline A & 1479 & 46.18 \\
SW & 171 & 5.34 \\
ASW & 151 & 4.71 \\
\hline
\end{tabular}



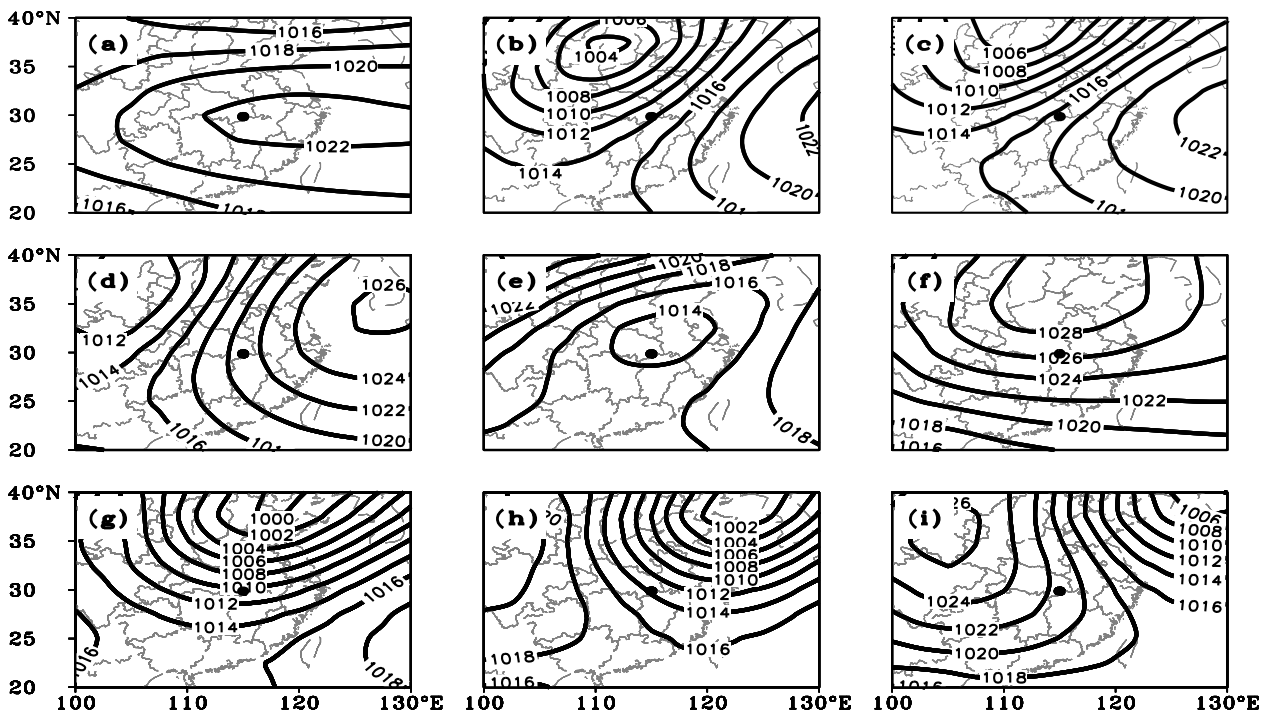

Fig. 2. Sea level pressure distribution of the major circulation patterns. (a) A type; (b) SW type; (c) ASW type; (d) AS type; (e) C type; (f) AE type; (g) W type; (h) NW type; (i) AN type. Contour interval is $1 \mathrm{hPa}$. The dot located in $115^{\circ} \mathrm{E}, 30^{\circ} \mathrm{N}$ indicates the location of Wuhan.

Table 3. Number of days and occurrence frequency of circulation patterns during $2016-2020$.

\begin{tabular}{ccc}
\hline Pattern & Days & Occurrence frequency [\%] \\
\hline A & 196 & 46.17 \\
C & 20 & 5.50 \\
SW & 16 & 5.24 \\
\hline
\end{tabular}

\section{Occurrence frequency of major circulation patterns during LDJA in 2020}

During LDJA in 2020, the most occurrence frequency of circulation patterns is A type (49.35\%, Fig. 2a), It is followed by C (9.09\%, Fig. 2e), W (6.49\%, Fig. 2 g) and NW (5.19\%, Fig. 2h) types. Compared with LDJA period in previous years (Table 2 and 3), the occurrence frequency of $\mathrm{C}, \mathrm{W}, \mathrm{AE}$, and NW types (Figs. 2e, g, f and h) increased, but AS and ASW (Figs. 2d and c) didn't occur.

As shown in table 4, the occurrence days of A, C, W and NW types' circulation pattern are more than those during LDJA of 1979-2020. The occurrence days of SW type's circulation pattern is slightly less than those during LDJA of 1979-2020. That means, without COVID-19 lockdown in Wuhan the air pollution could become more severe than normal compared with the same period of 1979-2020. However, all air pollutants except $\mathrm{O}_{3}$ decreased during LDJA in 2020. With more occurrence days of these dominant atmospheric circulation patterns, the number of polluted days could rise in Wuhan during early 2020. Nevertheless, this scenario didn't occur. The COVID-19 lockdown did improve the air quality in Wuhan during early 2020. 
Table 4. Number of days and occurrence frequency of circulation patterns during LDJA in 2020.

\begin{tabular}{cccccc}
\hline Pattern & $\begin{array}{c}\text { Days } \\
\text { during } \\
\text { LDJA, } \\
\mathbf{2 0 2 0}\end{array}$ & $\begin{array}{c}\text { Occurrence } \\
\text { frequency } \\
\text { during LDJA, } \\
\mathbf{2 0 2 0}[\%]\end{array}$ & $\begin{array}{c}\text { Mean }(\boldsymbol{\mu}) \text { of } \\
\text { days during } \\
\mathbf{1 9 7 9 - 2 0 2 0}\end{array}$ & $\begin{array}{c}\text { Standard } \\
\text { deviation }(\boldsymbol{\sigma}) \text { of } \\
\text { days during } \\
\mathbf{1 9 7 9 - 2 0 2 0}\end{array}$ & Interval \\
\hline A & 38 & 49.35 & 35.21 & 7.00 & $(\mu-\sigma, \mu+\sigma)$ \\
C & 7 & 9.09 & 3.00 & 2.52 & $(\mu-2 \sigma, \mu+2 \sigma)$ \\
W & 6 & 6.49 & 3.14 & 2.14 & $(\mu-2 \sigma, \mu+2 \sigma)$ \\
SW & 4 & 5.19 & 4.07 & 1.98 & $(\mu-\sigma, \mu+\sigma)$ \\
NW & 4 & 5.19 & 2.79 & 1.55 & $(\mu-2 \sigma, \mu+2 \sigma)$ \\
\hline
\end{tabular}

\section{Conclusions}

This study verified that the fact that the concentration of air pollutants decreased during COVID-19 lockdown in Wuhan. Then, Lamb-Jenkinson method was applied to classify the surface circulation patterns of Wuhan, and discuss the relationship between the improvement of air quality and the change of surface circulation situation.

All air pollutants except $\mathrm{O}_{3}$ decreased during LDJA in 2020. The occurrence days of A, C, W and NW types' circulation pattern during LDJA in 2020 are more than those during LDJA of 1979-2020. The occurrence days of SW type's circulation pattern is slightly less than those during LDJA of 1979-2020. The COVID-19 lockdown did improve the air quality in Wuhan during early 2020.

\section{Acknowledgments}

The special project "The impact of weather conditions on the spread of pandemic influenza virus (grant no. 2020xtzx004)" from Collaborative Innovation Center on Forecast and Evaluation of Meteorological Disasters, Nanjing University of Information Science and Technology.

\section{References}

[1] X. Yue, Y. Lei, H. Zhou, Z. Liu, L. Husi, Z. Cai, J. Lin, Z. Jiang, H. Liao, Trans Atmos. Sci. 43 (2020)

[2] C. Fan, Y. Li, J. Guang, Z. Li, A. Elnashar, M. Allam, G. Leeuw, Remote. Sens. 12 (2020)

[3] B. Chu, S. Zhang, J. Liu, Q. Ma, H. He, J. Environ. Sci. 99 (2020)

[4] X. Wang, R. Zhang, Bull. Am. Meteorol. Soc. 101 (2020)

[5] I. Sulaymon, Y. Zhang, P. Hopke, Y. Zhang, J. Hua, X. Mei, Atmos. Res. 250 (2021)

[6] A. AI-Hemoud, M. AI-Sudairawi, M. AI-Rashidi, W. Behbehani, A. AI-Khayat, Nat. Hazards. 97 (2019)

[7] E. Zhang, Y. Yang, X. Dong, Y. Yang, D. Nie, J. Hao, Meteorol. Environ. Res. 6 (2015) 
[8] M. Fikeraddis, L. Endeshaw, Environ. Earth. Sci. 02 (2020)

[9] Q. Zhao, W. Zhang, S. Wang, Plateau. Meteor. 22 (2003)

[10] B. Chen, Z. Lei, Q. Dong, J. Jiang, Y. Huo, Meteor. Environ. Sci. 40 (2017)

[11] Q. Li, F. Li, Y. Ye, S. Liu, J. Tro. Meteor. 15 (1999)

[12] X. Li, J. Yang, J. Ma, J. Wang, K. Zhao, Q. Ren, Y. Zhao, Meteor. 31 (2012)

[13] L. Chen, X. Zhi, J. Tan, H. Hu, Meteor. Mon. 42 (2016)

[14] M. Chang, W. Xiang, J. Qian, X. Zhang, J. Chen, X. Yang, G. Zhang, Acta. Sci. Circum. 40 (2020)

[15] G. Spellman, Theor. Appl. Climatol. 128 (2017)

[16] Ministry of Environmental Protection of the People's Republic of China Technical Regulation on Ambient Air Quality Index (on trial) (2012)

[17] B.Silver, C. Reddington, S. Arnold, D. Spracklen, Environ. Res. Lett. 13 (2018)

[18] X. Jin, T. Holloway, J. Geophys. Res. Atmos. 120 (2015)

[19] F. Huang, J. Zhou, H. Li, N. Chen, Res. Environ. Sci. 33 (2020)

[20] M. Li, K. Yuan, K. Hu, W. Liu, Torrential. Rain. Disaster. 38 (2019) 